\title{
Quasistationary quaternionic Hamiltonians and complex stochastic maps
}

\author{
G. Scolarici*and L. Solombrino ${ }^{\dagger}$ \\ Dipartimento di Fisica dell'Università del Salento \\ and INFN, Sezione di Lecce, I-73100 Lecce, Italy
}

August 9, 2021

\begin{abstract}
We show that the complex projections of time-dependent $\eta$-quasiantiHermitian quaternionic Hamiltonian dynamics are complex stochastic dynamics in the space of complex quasi-Hermitian density matrices if and only if a quasistationarity condition is fulfilled, i. e., if and only if $\eta$ is an Hermitian positive time-independent complex operator. An example is also discussed.
\end{abstract}

\section{Introduction}

Studies on non-Hermitian $\mathcal{P} \mathcal{T}$-symmetric (or, better, pseudo-Hermitian) Hamiltonians 1 proven that it is possible to formulate a consistent quantum theory based on such non-Hermitian Hamiltonians 2 at least whenever diagonalizable time-independent Hamiltonians having a real spectrum are taken into account. It was further shown that if the above hypotheses hold, complex quasi-Hermitian systems can be described as open systems, and a master equation was derived [3], proving that the evolution of such systems obeys a one-parameter semigroup law.

Moreover, the theory of open quantum systems can be obtained, in many relevant physical situations, as the complex projection of quaternionic closed quantum systems [4, [5, 6], 7]. In particular, it was shown that the complex projection of $\eta$-quasianti-Hermitian quaternionic time-independent Hamiltonian dynamics are ruled by one-parameter semigroups of maps in the space of complex quasi-Hermitian density matrices if and only if $\eta$ is an Hermitian positive complex operator [8], 9 .

In this paper, we will go more inside to this subject, by considering timedependent Hamiltonians. Such a problem was recently investigated [10] in the

*e-mail: scolarici@le.infn.it

†e-mail: solombrino@le.infn.it 
complex case, and a necessary and sufficient condition was derived for the unitarity of time evolution. We intend here to exploit an analogous method in the quaternionic case, on one hand; on the other hand, we will show that complex stochastic maps (in the sense by Sudarshan et al. [11) can be obtained by complex projection of time-dependent $\eta$-quasistationary quaternionic Hamiltonians.

This paper is organized as follows. In Sec. 2 we firstly recall some previous results on quaternionic pseudo-Hermitian density matrices and next we derive a necessary and sufficient condition for the $\eta$-unitarity of the time evolution associated with a $\eta$-pseudoanti-Hermitian quaternionic Hamiltonian. In Sec. 3 we restrict ourselves to consider positive operators $\eta$ only, and we define $\eta$-quasistationary quaternionic Hamiltonians. The dynamics ruled by such Hamiltonians are then investigated and explicitly written down, together with their complex projection which constitue stochastic maps in the space of the $\eta$-quasi-Hermitian complex density matrices. Such results are illustrated by an example in Sec. 4. Finally, in Sec. 5, we show how one can obtain the full class of $\eta$-quasistationary quaternionic dynamics, and construct the stochastic map associated with a generic time-dependent anti-Hermitian quaternionic operator.

\section{Pseudoanti-Hermitian quaternionic Hamilto- nian dynamics}

In this section, we will introduce the notion of quaternionic pseudo-Hermitian density matrix and a corresponding Liouville-von Neumann type equation will be derived.

Denoting by $O^{\ddagger}$ the adjoint of an operator $O$ with respect to the pseudo-inner product

$$
(\cdot, \cdot)_{\eta}=(\cdot, \eta \cdot)
$$

(where $(\cdot, \cdot)$ represent the standard quaternionic inner product in the space $\mathbb{Q}^{n}$ ), we have

$$
O^{\ddagger}=\eta^{-1} O^{\dagger} \eta
$$

so that for any $\eta$-pseudo-Hermitian operator, i. e., satisfying the relation,

$$
\eta O \eta^{-1}=O^{\dagger}
$$

one has, $O=O^{\ddagger}$.

If $O$ is $\eta$-pseudo-Hermitian, Eq. (3) immediately implies that $\eta O$ is Hermitian, so that the expectation value of $O$ in the state $|\psi\rangle$ with respect to the pseudo-inner product (11) can be obtained,

$$
\langle\psi|\eta O| \psi\rangle=\operatorname{Re} \operatorname{Tr}(|\psi\rangle\langle\psi| \eta O)=\operatorname{Re} \operatorname{Tr}(\tilde{\rho} O),
$$

where $\tilde{\rho}=|\psi\rangle\langle\psi| \eta$.

More generally, if $\rho$ denotes a generic quaternionic density (hence Hermitian and positive) matrix, we can associate with it a generalized density matrix $\tilde{\rho}$ by 
means of a one-to-one mapping in the following way:

$$
\tilde{\rho}=\rho \eta
$$

and obtain $\langle O\rangle_{\eta}=\operatorname{Re} \operatorname{Tr}(\tilde{\rho} O)$.

Note that $\tilde{\rho}$ is $\eta$-pseudo-Hermitian:

$$
\tilde{\rho}^{\dagger}=\eta \rho=\eta \tilde{\rho} \eta^{-1}
$$

As in the Hermitian case [4], [5, 6], 7], Eq. (4) immediately implies that the expectation value of an $\eta$-pseudo-Hermitian operator $O$ on the generalized state $\tilde{\rho}$ depends on the quaternionic parts of $O$ and $\tilde{\rho}$, only if both the operator and the generalized state are represented by genuine quaternionic matrices. Hence, if a $\eta$-pseudo-Hermitian operator $O$ is described by a complex matrix, its expectation value does not depend on the quaternionic part $j \tilde{\rho}_{\beta}$ of the state $\tilde{\rho}=\tilde{\rho}_{\alpha}+j \tilde{\rho}_{\beta}$.

It was shown that whenever the quaternionic Hamiltonian $H$ of a quantum system is $\eta$-pseudoanti-Hermitian, i. e.,

$$
\eta H \eta^{-1}=-H^{\dagger}
$$

where $\eta=\eta^{\dagger}$, the pseudo-inner product (11), is invariant under the time traslation generated by $H$ provided that $\eta$ does not depend on $t[8]$ :

$$
\langle\psi(t)|\eta| \psi(t)\rangle=\langle\psi(0)|\eta| \psi(0)\rangle .
$$

Denoting by $V(t)$ the evolution operator

$$
|\psi(t)\rangle=V(t)|\psi(0)\rangle
$$

Eq. (7) immediately implies

$$
V^{\dagger} \eta V=\eta
$$

i. e., $V$ is $\eta$-unitary. Whenever $H$ is time-independent, $\eta$-unitarity of $V$ is quite apparent, owing to its explicit form $V(t)=e^{-H t}(\hbar=1)$ and invoking $\eta$-pseudoanti-Hermiticity of $H$.

Moreover, from

$$
\rho(t)=V \rho(0) V^{\dagger}
$$

by easy calculations, we obtain for a generalized quaternionic density matrix $\tilde{\rho}$

$$
\tilde{\rho}(t)=V(t) \tilde{\rho}(0) V(t)^{-1} \text {. }
$$

In conclusion, $\eta$-unitarity of the time-evolution is a consequence of the $\eta$-pseudoantiHermiticity of $H$.

Conversely, let us assume unitarity of the time-evolution with respect to a (possibly time-dependent) $\eta$-inner product:

$$
\langle\psi(0)|\eta(0)| \phi(0)\rangle=\langle\psi(t)|\eta(t)| \phi(t)\rangle
$$


Then, this condition is equivalent to

$$
\eta(0)=V^{\dagger}(t) \eta(t) V(t)
$$

Differentiating both sides of the preceding equation we immediately get

$$
\left(\frac{d}{d t} \eta(t)\right) \eta^{-1}(t)=H^{\dagger}(t)+\eta(t) H(t) \eta(t)^{-1}
$$

where

$$
H(t)=-\left(\frac{d}{d t} V(t)\right) V^{-1}(t) .
$$

Eq. (12) shows that $H(t)$ is $\eta$-pseudoanti-Hermitian if and only if $\eta$ is timeindependent. In this case, the time evolution of $\tilde{\rho}(t)$ is described at finite level by Eq. (11) and at infinitesimal level by the usual Liouville-von Neumann equation:

$$
\frac{d}{d t} \tilde{\rho}(t)=-[H(t), \tilde{\rho}] .
$$

From Eq. (11), the conservation of the $\eta$-pseudo-norm immediately follows:

$$
\operatorname{Re} \operatorname{Tr} \tilde{\rho}(t)=\operatorname{Re} \operatorname{Tr} \tilde{\rho}(0) .
$$

From Eqs. (11), (9) and the $\eta$-pseudo-Hermiticity of $\tilde{\rho}(0)$ we immediately get

$$
\eta \tilde{\rho}(t) \eta^{-1}=\eta V(t) \tilde{\rho}(0) V^{-1}(t) \eta^{-1}=V^{\dagger-1}(t) \eta \tilde{\rho}(0) \eta^{-1} V^{\dagger}(t)=\tilde{\rho}^{\dagger}(t),
$$

i. e., $\tilde{\rho}(t)$ is $\eta$-pseudo-Hermitian.

\section{Quasistationary quaternionic Hamiltonian dy- namics and their complex projections}

In this section, we restrict ourselves to consider the space of quaternionic quasiHermitian density matrices, that is the subclass of $\eta$-pseudo-Hermitian density matrices where $\eta=T^{2}$ for some nonsingular bounded Hermitian operator $T$.

An important property of such generalized density matrices is that they are positive definite; indeed, putting $\eta=T^{2}$ into Eq. (5), from the positivity of $\rho$ we immediately obtain $T \tilde{\rho} T^{-1}=T \rho T=T \rho T^{\dagger} \geq 0[12$.

Then, the inner product (1) we introduced in the Hilbert space is positive, so that all the usual requirements for a proper quantum measurement theory can be maintained [13, 14, 15, 22. Hence, according to the discussion in Sec. 2,

the $\eta$-unitarity of the time-evolution implies that a time-dependent quaternionic Hamiltonian operator $H(t)$ defines a consistent unitary quaternionic quantum 
system if and only if $H(t)$ is $\eta$-pseudoanti-Hermitian for a time-independent positive $\eta$ operator. We will call such a Hamiltonian $\eta$-quasistationary [10].

When one considers $\eta$-quasistationary quaternionic dynamics, any $\eta$-unitary operator $V(t)$ can be decomposed as follows:

$$
V=T^{-1} U(t) T
$$

where $U^{\dagger} U=1$.

In fact, by using Eq. (9) and imposing unitarity, we immediately get

$$
T^{-1} V^{\dagger} T T V T^{-1}=\mathbf{1} .
$$

Recalling that $\eta$ is time-independent, we immediately obtain from Eqs. (15), (13)

$$
H(t)=T^{-1} \mathfrak{H}(t) T,
$$

where $\mathfrak{H}^{\dagger}(t)=-\mathfrak{H}(t)$

Let us denote by $M(\mathbb{Q})$ and $M(\mathbb{C})$ the space of $n \times m$ quaternionic and complex matrices respectively and let $M=M_{\alpha}+j M_{\beta} \in M(\mathbb{Q})$. We define the complex projection

$$
P: M(\mathbb{Q}) \rightarrow M(\mathbb{C})
$$

by the relation

$$
P[M]=\frac{1}{2}[M-i M i]=M_{\alpha} .
$$

In order to discuss the complex projection of quaternionic $\eta$-quasistationary dynamics, we recall the following properties [9]:

i) The complex projection $\tilde{\rho}_{\alpha}$ of a $\eta$-quasi-Hermitian quaternionic matrix $\tilde{\rho}=\tilde{\rho}_{\alpha}+j \tilde{\rho}_{\beta}$ is $\eta$-quasi-Hermitian if and only if the entries of $\eta$ are complex;

ii) The complex projection $\tilde{\rho}_{\alpha}$ of a $\eta$-quasi-Hermitian quaternionic matrix $\tilde{\rho}=\tilde{\rho}_{\alpha}+j \tilde{\rho}_{\beta}$ with a complex positive $\eta$, is positive and $\operatorname{Re} \operatorname{Tr} \tilde{\rho}_{\alpha}=1$.

We sketch here the proof of property ii). By property i) it is $\tilde{\rho}_{\alpha}=\rho_{\alpha} \eta$. Since $\eta=T^{2}$, from the positivity of $\rho_{\alpha}$ we immediately obtain

$$
T \tilde{\rho}_{\alpha} T^{-1}=T \rho_{\alpha} T^{\dagger} \geq 0,
$$

hence $\tilde{\rho}_{\alpha} \geq 0$. Furthermore, trivially, $\operatorname{Re} \operatorname{Tr} \tilde{\rho}_{\alpha}=\operatorname{Re} \operatorname{Tr} \tilde{\rho}=1$.

Now, let us consider a dynamics ruled by a $\eta$-quasistationary quaternionic Hamiltonian. Eq. (11) shows that this dynamics represent a mapping into the set of $\eta$-quasi-Hermitian quaternionic density matrices; moreover, if $\eta$ is complex and positive, properties i) and ii) ensure that the complex projection $\tilde{\rho}_{\alpha}$ of $\tilde{\rho}$ is a complex $\eta$-quasi-Hermitian density matrix for any $\tilde{\rho}$. Hence, we can conclude that the complex projection of a $\eta$-quasistationary quaternionic Hamiltonian dynamics, (with $\eta$ complex positive) is a complex stochastic dynamics in the space of $\eta$-quasi-Hermitian complex density matrices. 
The explicit form of such dynamics can be obtained from Eqs. (99 111) decomposing $V$ in terms of its complex parts $V_{\alpha}$ and $V_{\beta}: V=V_{\alpha}+j V_{\beta}$. Indeed by Eq. (10) one has

$$
\rho_{\alpha}(0) \rightarrow \rho_{\alpha}(t)=V_{\alpha} \rho_{\alpha}(0) V_{\alpha}^{\dagger}+V_{\beta}^{*} \rho_{\alpha}^{*}(0) V_{\beta}^{T}+V_{\alpha} \rho_{\beta}^{*}(0) V_{\beta}^{T}-V_{\beta}^{*} \rho_{\beta}(0) V_{\alpha}^{\dagger},
$$

which is a complex positive map in the space of Hermitian density matrices $\rho_{\alpha}$ ( $*$ and $T$ denote as usual complex conjugation and transposition, respectively). It follows that

$$
\tilde{\rho}_{\alpha}(t)=\left(V_{\alpha} \rho_{\alpha}(0) V_{\alpha}^{\dagger}+V_{\beta}^{*} \rho_{\alpha}^{*}(0) V_{\beta}^{T}+V_{\alpha} \rho_{\beta}^{*}(0) V_{\beta}^{T}-V_{\beta}^{*} \rho_{\beta}(0) V_{\alpha}^{\dagger}\right) \eta .
$$

Eq. (18) can be rewritten in term of $\widetilde{\rho}_{\alpha}(0)$ and $\widetilde{\rho}_{\beta}(0)$ putting $V^{-1}=W_{\alpha}+$ $j W_{\beta}$ and using the relations $V_{\alpha}^{\dagger} \eta=\eta W_{\alpha},-V_{\beta}^{T} \eta=\eta^{*} W_{\beta}$ (see Eq. (9))

$$
\widetilde{\rho}_{\alpha}(t)=V_{\alpha} \widetilde{\rho}_{\alpha}(0) W_{\alpha}-V_{\beta}^{*} \widetilde{\rho}_{\alpha}^{*}(0) W_{\beta}-V_{\alpha} \widetilde{\rho}_{\beta}^{*}(0) W_{\beta}-V_{\beta}^{*} \widetilde{\rho}_{\beta}(0) W_{\alpha} .
$$

At a infinitesimal level the previous equation get

$$
\frac{d}{d t} \widetilde{\rho}_{\alpha}=-\left[H_{\alpha}, \widetilde{\rho}_{\alpha}\right]+H_{\beta}^{*} \widetilde{\rho}_{\beta}-\widetilde{\rho}_{\beta}^{*} H_{\beta}
$$

where the symplectic decomposition of $H$ has been used.

It is worthwhile to stress that, unlike what happens whenever $H$ is timeindependent (in such case a one-parameter semigroup dynamics can always be associated with $H$ [9]), in the general case we considered here the evolution operator does not obey a semigroup law, as the example in the following section will show explicitly.

\section{An example}

Let us observe firstly that the more general 2-dimensional complex positive $\eta$ operator is given by

$$
\eta=T^{2}=\left(\begin{array}{cc}
x & z \\
z^{*} & y
\end{array}\right)\left(\begin{array}{cc}
x & z \\
z^{*} & y
\end{array}\right)=\left(\begin{array}{cc}
x^{2}+|z|^{2} & (x+y) z \\
(x+y) z^{*} & y^{2}+|z|^{2}
\end{array}\right)
$$

where $x, y \in \mathbb{R}, z \in \mathbb{C}$ and $x y \neq|z|^{2}$ and

$$
T^{-1}=\frac{1}{x y-|z|^{2}}\left(\begin{array}{cc}
y & -z \\
-z^{*} & x
\end{array}\right)
$$

The complex dynamical map we will study, is obtained as the complex projection of a deformation of the quaternionic unitary map:

$$
U(t)=\left(\begin{array}{cc}
\sqrt{1-(\sin 2 t)^{4}}+j e^{-i \theta}(\sin 2 t)^{2} & 0 \\
0 & 1
\end{array}\right), \theta \in \mathbb{R}
$$


to which corresponds the anti-Hermitian time-dependent Hamiltonian

$$
\mathfrak{H}(t)=-\left(\frac{d}{d t} U(t)\right) U^{\dagger}(t)=\left(\begin{array}{cc}
j \frac{-4 e^{-i \theta} \sin 2 t \cos 2 t}{|\cos 2 t| \sqrt{1+(\sin 2 t)^{2}}} & 0 \\
0 & 0
\end{array}\right) .
$$

We extensively studied such Hamiltonian [7, which generalizes to the quaternionic case a complex stochastic dynamics, arising in some decoherence modeling schemes [16].

From the previous two equations and Eqs. (16), (15) we get

$$
H(t)=T^{-1} \mathfrak{H}(t) T=j \frac{-4 e^{-i \theta} \sin 2 t \cos 2 t}{\left(x y-|z|^{2}\right)|\cos 2 t| \sqrt{1+(\sin 2 t)^{2}}}\left(\begin{array}{cc}
y x & y z \\
-z x & -z^{2}
\end{array}\right)
$$

and

$$
V(t)=T^{-1} U(t) T=\frac{1}{x y-|z|^{2}}\left(\begin{array}{cc}
y x q-|z|^{2} & y(q-1) z \\
-z^{*}(q-1) x & -z^{*} q z+x y
\end{array}\right)
$$

where, $q=\sqrt{1-(\sin 2 t)^{4}}+j e^{-i \theta}(\sin 2 t)^{2}$.

Let the initial "pure" state be

$$
\tilde{\rho}(0)=\frac{1}{2}\left(\begin{array}{ll}
1 & 0 \\
0 & 1
\end{array}\right)+\frac{1}{2\left(x y-|z|^{2}\right)} j\left(\begin{array}{cc}
-(x+y) z^{*} e^{-i \theta} & -\left(|z|^{2}+y^{2}\right) e^{-i \theta} \\
\left(|z|^{2}+x^{2}\right) e^{-i \theta} & (x+y) z e^{-i \theta}
\end{array}\right)
$$

according with Eq. (11) the final state reads

$$
\begin{aligned}
\tilde{\rho}(t)= & \frac{1}{2}\left(\begin{array}{cc}
1 & 0 \\
0 & 1
\end{array}\right)+\frac{(\sin 2 t)^{2}}{2\left(x y-|z|^{2}\right)}\left(\begin{array}{cc}
y z^{*}-x z & y^{2}-z^{2} \\
\left(x^{2}-z^{* 2}\right) & -y z^{*}+x z
\end{array}\right)+ \\
& j \frac{e^{-i \theta} \sqrt{1-(\sin 2 t)^{4}}}{2\left(x y-|z|^{2}\right)}\left(\begin{array}{cc}
-z^{*}(x+y) & -|z|^{2}-y^{2} \\
|z|^{2}+x^{2} & z(x+y)
\end{array}\right) .
\end{aligned}
$$

The complex projection stochastic dynamics is given by

$$
\frac{1}{2}\left(\begin{array}{ll}
1 & 0 \\
0 & 1
\end{array}\right) \rightarrow \frac{1}{2}\left(\begin{array}{ll}
1 & 0 \\
0 & 1
\end{array}\right)+\frac{(\sin 2 t)^{2}}{2\left(x y-|z|^{2}\right)}\left(\begin{array}{cc}
y z^{*}-x z & y^{2}-z^{2} \\
\left(x^{2}-z^{* 2}\right) & -y z^{*}+x z
\end{array}\right)
$$

Note that the semigroup composition law does not hold. In fact, by a direct computation it is easy to verify that

$$
P\left[V(t) \tilde{\rho}(0) V^{-1}(t)\right] \neq P\left[V\left(t-t^{\prime}\right) V\left(t^{\prime}\right) \tilde{\rho}(0) V^{-1}\left(t^{\prime}\right) V^{-1}\left(t-t^{\prime}\right)\right],
$$

indeed,

$$
P\left[\left(V(t) \tilde{\rho}(0) V^{-1}(t)\right)_{21}\right]=(\sin 2 t)^{2}
$$

while 


$$
\begin{aligned}
& P\left[\left(V\left(t-t^{\prime}\right) V\left(t^{\prime}\right) \tilde{\rho}(0) V^{-1}\left(t^{\prime}\right) V^{-1}\left(t-t^{\prime}\right)\right)_{21}\right] \\
= & \left(\cos 2\left(t-t^{\prime}\right)\right)^{2}\left(\sin 2 t^{\prime}\right)^{2}-\left[\left(1-\left(\cos 2\left(t-t^{\prime}\right)\right)^{4}\right)\left(1-\left(\sin 2 t^{\prime}\right)^{4}\right)\right]^{\frac{1}{2}} .
\end{aligned}
$$

\section{Final remark}

Let a Hermitian nonsingular quaternionic operator $\eta$ be given. Then, the more general $\eta$-pseudoanti-Hermitian quaternionic Hamiltonian $H$ can be written in the following way [9]:

$$
H=F \eta,
$$

where $F^{\dagger}=-F$.

This peculiarity can be useful to obtain the full class of $\eta$-quasistationary quaternionic dynamics. In fact, for any time-dependent anti-Hermitian quaternionic operator $F(t)$, we can construct a corresponding anti-Hermitian operator $\mathfrak{H}(t)=\eta^{\frac{1}{2}} F(t) \eta^{\frac{1}{2}}$, and from Eq. (16) we can state that

$$
H(t)=\eta^{-\frac{1}{2}} \mathfrak{H}(t) \eta^{\frac{1}{2}},
$$

is a $\eta$-quasistationary Hamiltonian, and construct the stochastic (complex) map associated with it, by the methods used in the example above. Conversely, let a $\eta$-quasistationary quaternionic dynamics be given, then, a time-dependent anti-Hermitian operator $F(t)$ can be associated with it.

Acknowledgements One of us, G. S., wishes to thank prof. M. Znojil for useful discussions and financial support during a short stay in Prague.

\section{References}

[1] Proceedings of the Ist, IInd, IIIrd, IVth and Vth International Workshops on "Pseudo-Hermitian Hamiltonians in Quantum Physics" in Czech. J. Phys. 54 (2004), Nos. 1, 10, Czech. J. Phys. 55 (2005), J. Phys. A 39 (2006) and Czech. J. Phys. 56 (2006), respectively.

[2] A. Mostafazadeh, J. Phys. A 36 (2003) 7081.

[3] G. Scolarici and L. Solombrino, Czech. J. Phys. 56 (2006) 935.

[4] G. Scolarici and L. Solombrino "Complex entanglement and quaternionic separability" in The Foundations of Quantum Mechanics: Historical Analysis and Open Questions-Cesena 2004, C. Garola, A. Rossi and S. Sozzo eds. (World Scientific, Singapore, 2006).

[5] M. Asorey and G. Scolarici, J. Phys. A 39 (2006) 9727. 
[6] M. Asorey, G. Scolarici and L. Solombrino, Theor. Math. Phys. 151 (2007) 733.

[7] M. Asorey, G. Scolarici and L. Solombrino, Phys. Rev. A 76 (2007) 12111.

[8] G. Scolarici, J. Phys. A 35 (2002) 7493.

[9] G. Scolarici, SIGMA 3 (2007) 088.

[10] A. Mostafazadeh, Phys. Lett. B 650 (2007) 208.

[11] P. M. Mathews, J. Rau and E. C. G. Sudarshan, Phys. Rev. 121 (1961) 920.

[12] F. Zhang, Lin. Alg. Appl. 251 (1997) 21.

[13] A. Blasi, G. Scolarici and L. Solombrino, J. Math. Phys. 46 (2005) 42104.

[14] F. G. Sholtz, H. B. Geyer and F. J. W. Hahne, Ann. Phys. 213 (1992) 74.

[15] A. Blasi, G. Scolarici and L. Solombrino, J. Phys. A 37 (2004) 4335.

[16] C. A. Rodriguez, A. Shaji and E. C. G. Sudarshan "Dynamics of Two Qubits: Decoherence and Entanglement Optimization Protocol" arXiv: quant-ph/0504051. 